\title{
Pediatric Post-Operative Atrio-Ventricular Block Meets the Affordable Care Act: A New Strategy for Management
}

\author{
Melissa L. Morello', Joan S. Steinberg ${ }^{2}$, Christopher Snyder ${ }^{1 *}$ \\ ${ }^{1}$ Division of Pediatric Cardiology, Rainbow Babies \& Children's Hospital, Case Western \\ Reserve University School of Medicine, Cleveland, OH, USA \\ ${ }^{2}$ School of Public Health and Tropical Medicine, Tulane University, New Orleans, LA, USA \\ Email: *Christopher.Snyder@uhhospitals.org
}

How to cite this paper: Morello, M.L., Steinberg, J.S. and Snyder, C. (2017) Pediatric Post-Operative Atrio-Ventricular Block Meets the Affordable Care Act: A New Strategy for Management. Open Journal of Pediatrics, 7, 118-127.

https://doi.org/10.4236/ojped.2017.73015

Received: June 2, 2017

Accepted: August 1, 2017

Published: August 4, 2017

Copyright (C) 2017 by authors and Scientific Research Publishing Inc. This work is licensed under the Creative Commons Attribution International License (CC BY 4.0).

http://creativecommons.org/licenses/by/4.0/

\begin{abstract}
Introduction: Post-operative (post-op) complete atrio-ventricular heart block (CAVB) occurs after $1 \%-4 \%$ of pediatric cardiac operations. Current practice dictates implantation of permanent pacemaker (PPM) when post-op CAVB persists $>9$ days. We propose that earlier PPM implantation may be the most cost-effective methodology since patient costs increase with extended length of stay (LOS). Methods: Data on the probabilities of persistent post-op CAVB were extracted from published reports. No individual patient data were utilized during this study. This was utilized to create a decision-making model and a total cost analysis on post-op day 0 - 10 to determine the most cost-efficient day to implant a PPM. Cost variables included estimates of daily cardiac ICU care, cost of PPM implantation, LOS, cost related to possible superficial or deep infection based on published prevalence rates $(2.3 \%$ and $4.9 \%$, respectively) and need for explant due to deep infection or recovery of native conduction. The model assumes 5-day minimum LOS and 1 day increase in LOS with PPM implantation. Cost data were obtained from relevant billing codes and manufacturer list prices for PPM and leads. A secondary analysis evaluated probability of unnecessary PPMs implanted and excess costs. Results: Post-op day (POD) 4 is the lowest total cost of PPM implantation for post-op CAVB, even when accounting for possible risk of either superficial or deep infection. A one-way sensitivity analysis accounting for variability of cardiac ICU care costs between centers ranging from $\$ 3000$ - $\$ 9000$ per day consistently replicates POD 4 as the most cost-effective day for PPM implantation. Implant on POD 4 results in a $26 \%$ chance of unnecessary implantation. Conclusions: The most cost-efficient day for PPM implantation for post-op CAVB is post-op day 4 , which results in a minimum total cost savings of
\end{abstract}


$\$ 17,422$ per patient. Added costs due to risk of superficial or deep infection are marginal due to low prevalence of post-operative infection in this population.

\section{Keywords}

Pediatric, Pacemaker, Post-Operative, Heart Block, Atrioventricular Block, Cost

\section{Introduction}

Healthcare costs have become an issue at the forefront of the US political economy in recent years. Current legislation such as The Patient Protection and Affordable Care Act (PPACA), enacted by President Barack Obama in 2010, and subsequent development of Accountable Care Organizations (ACOs) reflect a trend towards high-quality yet cost-efficient care. Advancements in surgical techniques, perfusion strategies and postoperative management in congenital heart disease (CHD) have decreased the incidence of post-operative (post-op) complete atrioventricular block (CAVB) from about $10 \%$ to $1 \%-4 \%$ in recent years [1] [2] [3]. However, a concurrent increase in complex surgeries for CHD reflects an increase in number of pediatric patients requiring permanent pacemaker (PPM) implantation [4]. CAVB is a Class I indication for PPM placement according to the revised ACC/AHA/HRS 2008 guidelines. Current practice reflects literature recommendations to implant a PPM when post-op CAVB persists beyond 9 days, as $97 \%$ of patients who recover their atrioventricular (AV) conduction do so by day 9 [3]. Post-op CAVB remains a complication that increases costs due to increased length of stay (LOS) and PPM implantation when CAVB does not recover [1]. However, given the significant costs accrued with each post-operative day (POD) in the hospital, we propose that earlier PPM implantation may be more cost-efficient in the immediate post-operative period.

This study presents a cost-efficiency analysis of ICU observation for recovery of post-op CAVB versus PPM implantation on post-op days 0 - 10 using previously published data on the probability of post-op CAVB during this time period and published costs for daily cardiac ICU monitoring and PPM implantation. Secondary analyses sought to establish excess costs related to probability of unnecessary PPM implantation and probability of post-operative superficial or deep pocket infection within the immediate post-op period.

While there have been previously published reports on the costs related to post-operative CAVB in patients with CHD in the US [1], this study is the first to describe the cost-efficiency of earlier PPM implantation in pediatric patients with post-op CAVB.

\section{Methods}

Data on the probabilities of persistent post-op CAVB were merged with cost da- 
ta to create a mathematical model that calculated the minimum total cost of PPM implantation on each POD from 0 - 10. A secondary mathematical model calculated the probability of excess PPM implantation based upon probability of $\mathrm{AV}$ conduction recovery, and these data were used to calculate excess costs related to unnecessary PPM implantation. Prevalence rates of both post-operative superficial and deep infection within the immediate post-operative period were merged with costs related to these complications and superimposed upon the previous two models to calculate excess costs related to infection. No individual patient data was utilized during this study.

Microsoft Excel (c) 2008 was utilized for all calculations.

\subsection{Model Assumptions}

The minimum total cost model assumes a 5-day LOS, regardless of intervention. LOS increases by 1 day if a PPM is implanted. The Excess PPM model assumes that the probability of $\mathrm{AV}$ conduction recovery is the difference between the probabilities of persistent post-op CAVB on each POD and POD 10. The Infection Cost model assumes: for superficial infection, medical floor LOS of 2 days and for deep infection, medical floor LOS of 3 days and cardiac intensive care unit LOS of 2 days. With either infection, the remainder of the $10-14$ day IV antibiotic course is assumed to be given at a remote site.

\subsection{Cost Variables}

Cost variables for the minimum total cost model included estimates of daily cardiac ICU care, cost of temporary epicardial lead placement and testing, cost of PPM implantation, PPM system (generator and leads), PPM testing and length of stay. Cost variables for the Excess PPM model included cost of PPM system, PPM implantation, PPM testing and PPM explant due to recovery of native conduction. Finally, cost variables related to superficial infection included LOS and peripherally inserted central catheter (PICC) line insertion for intravenous antibiotics. Cost variables for deep infection included LOS, PICC line insertion for intravenous antibiotics, cost of explant and cost of re-implantation.

\subsection{Data Sources}

Patients with persistent post-op CAVB

Data on the probability of persistent post-op CAVB were extracted from a previously published retrospective analysis of 54 pediatric patients $<21$ years of age with CHD who developed post-op CAVB at a major CHD center in the United States, with a follow-up period of 30 days after CHD surgery [3]. This report was used because it informs the current practice of PPM implantation if post-op CAVB persists beyond POD 9.

Patients with PPM-related Infections

Probabilities of PPM infections in pediatric patients were based on previously published prevalence rates of $2.3 \%$ for deep infection and $4.9 \%$ for superficial 
infection in a retrospective, single-center study of 267 patients with a mean age of 8.4 \pm 6.2 years followed for a median of 29.4 months from 1982-2001 [5].

\section{Cost data}

Cost data were obtained from comparing relevant billing codes with Medicare reimbursement tables published between 2012-2013 and manufacturer list prices for PPM systems published in the USA between 2012-2013. The cost of observation, including epicardial pacing wire insertion, was summed from recently published data on average CVICU costs for observation [6] [7] plus the cost of epicardial pacing wire insertion $(\$ 131$, billing code 33,210) [8]. The cost of intervention was the cost of observation plus the cost of PPM device, lead, insertion ( $\$ 497$, billing code 33,207 ) and interrogation $(\$ 38$, billing code 93,288 ) [8]. The assumed PPM to be used was either the Medtronic Sensia SR device (\$8000) [9] or the St. Jude Microny II SR + device (\$7395) [10], with a Bipolar lead (\$1400) [10]. The cost of superficial infection was the cost of 2 day LOS on a medical floor and insertion of a PICC line (total \$485, billing codes 36,569, 76,942 and 71,010) [11] for IV antibiotic administration. The cost of deep infection was the cost of 3 day LOS on a medical floor, insertion of a PICC line, PPM explant (\$1485, billing code 33,233) [11], 2 day LOS in the cardiac intensive care unit and PPM re-implantation after completion of IV antibiotic course.

\section{Results}

The minimum total cost analysis calculates the minimum total cost to the medical system for each patient with post-operative CAVB, assuming PPM implantation on each POD 0 - 10 and possible risk of either superficial or deep infection. POD 4 is the day that results in the lowest total cost of PPM implantation for persistent post-op CAVB, which results in $\$ 17,422$ of savings per patient in this cohort with a PPM is implanted on POD 4 versus waiting until POD 10 (Table 1). A one-way sensitivity analysis accounting for variability of cardiac ICU care costs between centers ranging from $\$ 3000$ - $\$ 9000$ per day consistently replicated POD 4 as the most cost-effective day for PPM implantation. Figure 1 and Figure 2 graphically represent the declining probability of post-operative CAVB on each POD versus the minimum total cost to the medical system for each patient on each POD.

Table 2 shows the probability of excess PPM implantation for each POD and the excess cost to each patient in the cohort in comparison to the difference in cost of observation and then implantation on POD 10. Implantation on POD 4 confers a $26 \%$ chance of inserting a PPM in a patient who may have otherwise recovered their native $\mathrm{AV}$ conduction, and this results in approximately 1 extra device-related infection (0.322 extra superficial infections and 0.686 deep infections), with the excess cost to each patient in the cohort totaling $\$ 2512$. This excess cost due to unnecessary PPM implantation and risk of infection reduces the total savings per patient in the cohort from $\$ 17,422$ to $\$ 14,910$ (14\%) when the PPM implantation occurs on POD 4. 


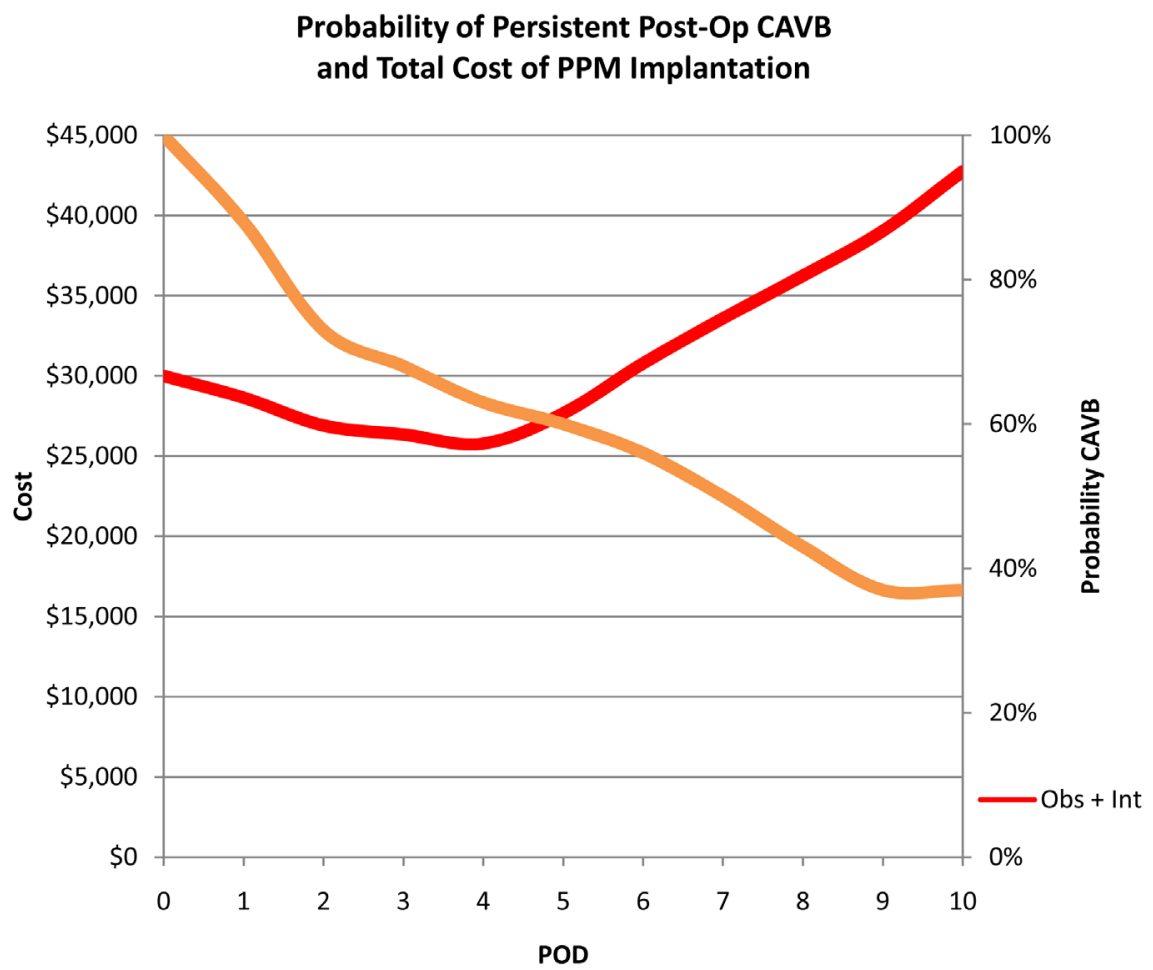

Figure 1. Cost comparison between patients with post-op CHB who are either observed for recovery of native conduction (orange line) or who have a PPM implanted on the corresponding POD due to persistence of CAVB (red line), from POD 0 - 10. CAVB = complete atrioventricular block; $\mathrm{PPM}=$ permanent pacemaker; $\mathrm{POD}=$ post-operative day.

Probability of Persistent Post-Op CAVB and Total Cost of PPM Implantation

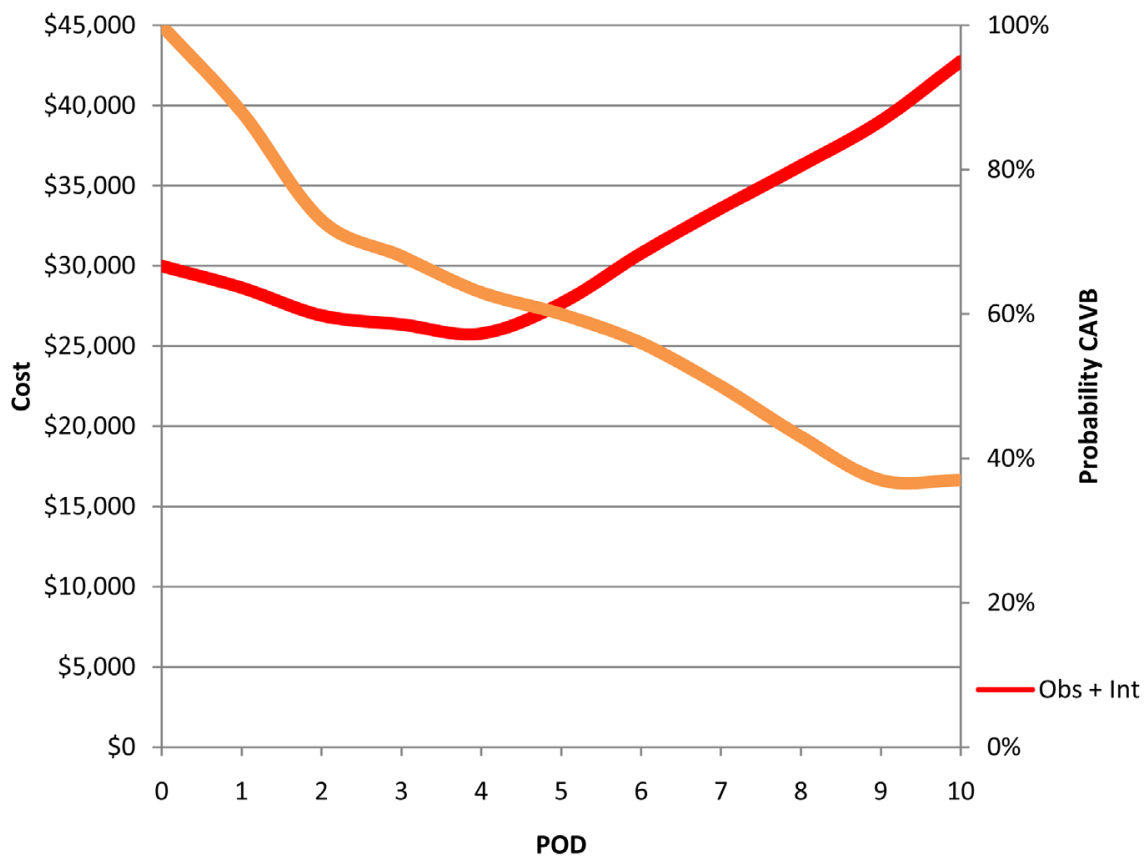

Figure 2, Post-Operative day. 
Table 1. Cost of PPM placement for persistent CAVB from POD 0 - 10.

\begin{tabular}{ccc}
\hline POD & Probability post-op CAVB & Minimum Total Cost \\
\hline 0 & 1 & $\$ 28,142$ \\
1 & 0.88 & $\$ 27,023$ \\
2 & 0.73 & $\$ 25,623$ \\
3 & 0.68 & $\$ 25,157$ \\
4 & 0.63 & $\$ 24,690$ \\
5 & 0.6 & $\$ 26,628$ \\
6 & 0.56 & $\$ 29,803$ \\
7 & 0.5 & $\$ 32,717$ \\
8 & 0.43 & $\$ 35,501$ \\
9 & 0.37 & $\$ 38,416$ \\
10 & 0.37 & $\$ 42,112$ \\
\hline
\end{tabular}

Model assumes that only patients with the probability of persistent CAVB receive PPMs. CAVB $=$ complete atrioventricular block; PPM = permanent pacemaker.

Table 2. Predicted excess costs of pacemaker implantation earlier than POD 9.

\begin{tabular}{cccc}
\hline POD & Excess PPM installed & $\begin{array}{c}\text { Cost of additional infections } \\
\text { based on excess PPM installed }\end{array}$ & $\begin{array}{c}\text { Excess cost of } \\
\text { PPM + infection }\end{array}$ \\
\hline 0 & 0.63 & $\$ 209.53$ & $\$ 6087.43$ \\
1 & 0.51 & $\$ 169.62$ & $\$ 4927.92$ \\
2 & 0.36 & $\$ 119.73$ & $\$ 3478.53$ \\
3 & 0.31 & $\$ 103.10$ & $\$ 2995.40$ \\
4 & 0.26 & $\$ 86.47$ & $\$ 2512.27$ \\
5 & 0.23 & $\$ 76.49$ & $\$ 3072.47$ \\
6 & 0.19 & $\$ 63.19$ & $\$ 2538.13$ \\
7 & 0.13 & $\$ 43.23$ & $\$ 1736.61$ \\
8 & 0.06 & $\$ 19.95$ & $\$ 801.51$ \\
9 & 0 & 0 & 0 \\
10 & 0 & 0 & 0 \\
\hline
\end{tabular}

Model accounts for probability of persistent CAVB on each POD and for probabilities of either superficial or deep infection. $\mathrm{PPM}=$ pacemaker; $\mathrm{POD}=\mathrm{p}$.

\section{Discussion}

This analysis evaluates the minimum total cost savings of earlier PPM implantation for post-op CAVB compared to the current practice of observation and PPM implantation when post-op CAVB persists beyond POD 9. While earlier implantation results in a savings of $\$ 17,422$ per patient in the cohort, it also results in unnecessary placement of PPMs in patients who would have otherwise recovered their conduction if observed until POD 10. However, even if patients recover their native conduction by POD 10, late recurrence of high-grade post- 
op AVB (Mobitz II or complete AV block) remains a risk, with a previously reported incidence of at least $0.3 \%-0.7 \%$ [12], but up to $5 \%-9 \%$ [3] [13], depending on type of CHD surgery and location and degree of conduction system injury. Excess costs incurred to the medical system due to unnecessary PPMs implanted on POD 4 and excess superficial or deep infections total \$2512, which only decreases the minimum total cost savings to each patient by $14 \%$. While earlier implantation of a PPM is more cost-efficient, in practice it may not be appropriate for all patients with post-op CAVB. Post-op CAVB occurs more often in patients undergoing operations involving bypass (3\% versus $2 \%$ of all CHD operations), Trisomy 21 and any surgery involving the AV canal [3], possibly making earlier implantation more plausible in these patients at higher risk of persistent post-op CAVB. However, because device-related infection is higher in patients with CHD and Trisomy 21 [5] [14], the risk of such adverse outcomes must be balanced with their respective costs compared to the cost of increased LOS for extended observation and any secondary hospital-acquired infections resulting from extended LOS. In addition, current practice aims to reduce the risk of device-related infections with administration of pre-operative prophylactic IV antibiotics. While no prospective randomized trials have examined primary prophylaxis for the prevention of device-related infection, current recommendations are to administer prophylactic antibiotics in all patients who are to receive electrophysiological cardiac devices such as PPMs or cardioverter-defibrillators for at least 1 dose 1 hour prior to implant and for 24 hours after wound closure [2].

Currently, the PPACA policies outline an infrastructure mainly for the practice of adult medicine, likely due to overall higher incidence of chronic illness in this population. Using provisions established by the PPACA, preliminary initiatives to include pediatric medicine in the development of ACOs include the Pediatric Demonstration Project, which calls for participating state Medicaid programs to allow pediatric medical providers to form an ACO and receive incentive payments based on quality guidelines established by the Department of Health and Human Services (DHHS). As we look forward to the implementation of such policies within the field of pediatric cardiology, models of cost-efficient care will help inform medical decision-making as providers assimilate their practice into this new era.

\section{Limitations}

This model does not include costs of possible adverse outcomes in the immediate post-operative period, including but not limited to pneumothorax, hemothorax, bleeding, migration, malfunction and need for explant due late conduction recovery $>30$ days post-implantation, though these complications have been previously described [13] [14]. Cost variability between centers will alter the dollar amounts reported in this study, but should not affect the overall trend. Further analysis is warranted to evaluate total lifetime costs related to unneces- 
sary PPM implantation in patients who would have otherwise recovered their native AV conduction before POD 10, including costs of device-related outpatient follow-up, routine device testing, and need for partial or total system replacement due to malfunction, migration, lead fracture or infection.

\section{Conclusion}

Given the additive daily costs associated with monitoring for recovery of conduction until POD 9, the most cost-efficient day for PPM implantation for postop CAVB is POD 4, which results in a minimum total cost savings of $\$ 17,422$ per patient. Added costs due to the $26 \%$ risk of unnecessary PPM implantation and extra superficial or deep infections due to unnecessary PPM implantation are marginal in comparison to the savings with earlier implantation, likely due to the overall low prevalence of device-related infection, even in patients with CHD. As more patients with CHD are living into adulthood than ever before, earlier PPM placement may confer a benefit to those who experienced transient post-op CAVB before POD 10 but had late recurrence of a high-grade AV block, which is a well-established risk. We believe that we have made a strong case for discussion regarding the "early" implantation of these devices in certain patients especially those with higher risk of permanent AV block after surgery.

\section{Author Contributions and Acknowledgements}

Melissa Morello, MD was responsible for background research, data collection and analysis and drafting of article.

Joan Steinberg, PhD provided significant assistance with the statistical model and design.

Christopher Snyder, MD was responsible for critical revision of article and approval of final submission.

A special thanks to Stephen Dreyer, MD for his expertise in Microsoft Excel @.

\section{Disclosures}

No financial disclosures.

\section{References}

[1] Anderson, J.B., Czosek, R.J., Knilans, T.K., Meganathan, K. and Heaton, P. (2012) Postoperative Heart Block in Children with Common Forms of Congenital Heart Disease: Results from the KID Database. Journal of Cardiovascular Electrophysiology, 23, 1-6. https://doi.org/10.1111/j.1540-8167.2012.02385.x

[2] Baddour, L., Bettman, M., Bolger, A., et al. (2003) Nonvalvular Cardiovascular Device-Related Infections. Circulation, 108, 2015-2031. https://doi.org/10.1161/01.CIR.0000093201.57771.47

[3] Weindling, S.N., Saul, P.J., Gamle, W.J., Mayer, J.E., Wessel, D. and Walsh, E.P. (1998) Duration of Complete Atriventricular Block after Congenital Heart Disease Surgery. American Journal of Cardiology, 82, 525-527. https://doi.org/10.1016/S0002-9149(98)00375-0 
[4] Welisch, E., Cherlet, E., Crespo-Martinez, E. and Hansky, B. (2010) A Single Institution Experience with Pacemaker Implantation in a Pediatric Population over 25 Years. Pacing \& Clinical Electrophysiology Pace, 33, 1112-1118. https://doi.org/10.1111/j.1540-8159.2010.02781.x

[5] Cohen, M.I., Bush, D.M., Gaynor, J.W., Vetter, V.L., Tanel, R.E. and Rhodes, L.A. (2002) Pediatric Pacemaker Infections: Twenty Years of Experience. Journal of Thoracic and Cardiovascular Surgery, 124, 821-827. https://doi.org/10.1067/mtc.2002.123643

[6] Chalom, R., Raphaely, R.C. and Costarino, A.T.J. (1999) Hospital Costs of Pediatric Intensice Care. Critical Care Medicine, 27, 2079-2085. https://doi.org/10.1097/00003246-199910000-00001

[7] Edwards, J.D., Rivanis, C., Kun, S.S., Caughey, A.B. and Keens, T.G. (2011) Costs of Hospitalized Ventilator-Dependent Children: Differences between a Ventilator Ward and Intensive Care Unit. Pediatric Pulmonology, 46, 356-361. https://doi.org/10.1002/ppul.21371

[8] Medtronic (2013) Single and Dual Chambered Pacemakers. Price List Effective February 2013. Medtronic, Atlanta.

[9] Medrad (2013) Cost of Epicardial Wire Placement. Medrad, Warrendale. http://www.medrad.com/en-us/resources/KnowledgeCenter/Documents/3087-Q1-3 2013_Reimbursement_Broch.pdf

[10] St. Jude Medical (2012) Cardiac Rhythm Management Products. Price List Effective July 2012. St. Jude Medical, Little Canada.

[11] Biotronik (2013) 2013 Medicare Physician Fee Schedule Update. 2012 and 2013 Final Medicare Physician Fee Schedule Regulations. http://www.biotronik.com/

[12] Klug, D., Vaksmann, G., Jarwe, M., Wallet, F., Francart, C. and Kacet, R.C. (2003) Pacemaker Lead Infection in Young Patients. Pacing \& Clinical Electrophysiology Pace, 26, 1489-1493. https://doi.org/10.1046/j.1460-9592.2003.t01-1-00215.x

[13] Lin, A., Mahle, W.T., Frias, P.A., Fischbach, P.S., Kogon, B.E., Kanter, K.R. and Kirshbom, P.M. (2010) Early and Delayed Atrioventricular Conduction Block after Routine Surgery for Congenital Heart Disease. Journal of Thoracic and Cardiovascular Surgery, 140, 158-160. https://doi.org/10.1016/j.jtcvs.2009.12.050

[14] Silvetti, M., Drago, F., Marcora, S. and Rava, L. (2007) Outcome of Single-Chamber, Ventricular Pacemakers with Transvenous Leads Implanted in Children. Europace, 9, 894-899. https://doi.org/10.1093/europace/eum164 


\section{Abbreviations}

Post-op $=$ Post-operative

CAVB = Complete Atrioventricular Block

$\mathrm{PPM}=$ Permanent Pacemaker

LOS $=$ Length Of Stay

POD $=$ Post-Operative Day

PPACA = Patient Protection and Affordable Care Act

ACO = Accountable Care Organization

$\mathrm{CHD}=$ Congenital Heart Disease

$\mathrm{AV}=$ Atrioventricular

PICC $=$ Peripherally Inserted Central Catheter

DHHS $=$ Department of Health and Human Services

Submit or recommend next manuscript to SCIRP and we will provide best service for you:

Accepting pre-submission inquiries through Email, Facebook, LinkedIn, Twitter, etc. A wide selection of journals (inclusive of 9 subjects, more than 200 journals) Providing 24-hour high-quality service User-friendly online submission system Fair and swift peer-review system Efficient typesetting and proofreading procedure Display of the result of downloads and visits, as well as the number of cited articles Maximum dissemination of your research work

Submit your manuscript at: http://papersubmission.scirp.org/ Or contact ojped@scirp.org 